\title{
Modelling strategies to predict hospital demand during the COVID-19 outbreak in Bogota, Colombia
}

Claudia Rivera-Rodriguez ( $\square$ clriverarodriguez@gmail.com )

University of Auckland https://orcid.org/0000-0002-9614-7036

\section{Beatriz Piedad Urdinola}

Universidad Nacional de Colombia Facultad de Ciencias

Research article

Keywords: Covid-19; SEIR models; Bogot】a-Colombia

Posted Date: May 11th, 2020

DOI: https://doi.org/10.21203/rs.3.rs-23344/v1

License: (9) This work is licensed under a Creative Commons Attribution 4.0 International License.

Read Full License 


\title{
Modelling strategies to predict hospital demand during the COVID-19 outbreak in Bogotá, Colombia
}

\author{
Claudia Rivera-Rodriguez ${ }^{1 *}$ and Beatriz Piedad Urdinola ${ }^{1,2}$
}

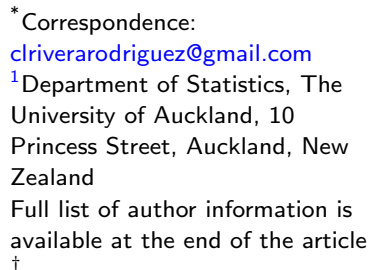

\begin{abstract}
Background: Colombia, like many developing nations, does not have a strong health system able to respond to a pandemic of the magnitude of Covid-19. There is an increasing need to create a model that allows particular clinics and hospitals to the number of patients that require Intensive Care Units-ICU care (critical), and the number of patients that require hospital care (severe), but not ICU care.

Methods: This paper presents a prediction of the total number of ICU and regular beds that will be needed during the pandemic COVID-19 for Bogotá-Colombia. We use a SEIR model that includes three different compartments of infection: those who can stay at home, those in regular hospital beds and those in need of ICU treatment. The model allows for a time varying transmission rate which we use to incorporate the measures introduced by the government over the period of one year.
\end{abstract}

Results: The model predicts that by mid July 2020, the city will reach the peak of the epidemic with a total of 22526 prevalent ICUs needed and 84816 regular hospital beds needed. By the end of May 2020, the number of patients that need ICUs will overpass the current capacity set at 2000 beds for ICU hospital beds in the city. The model predicts that the death toll by the same date will reach 1752 people and the number of cases will be 54652 inhabitants by then. We provide a Shiny app available in https://claudia-rivera-rodriguez.shinyapps.io/shinyappcovidclinic/. The original values in the app reproduce the results of this paper, but the parameters and starting values can be changed according to the users needs.

Conclusions: COVID-19 has posed too many challenges to health systems around the globe, this model is an useful tool for cities, hospitals and clinics in Colombia that need to prepare for the excess demand of services that a pandemic like this one generates. Unfortunately, the model predicts that by July capacity of the system in Bogotá will not be enough. We expect the lock-down rules strength in the future days, so the death toll is not as bad as predicted by this model.

Keywords: Covid-19; SEIR models; Bogotá-Colombia

\section{Background}

The novel coronavirus disease 2019 (COVID-19) epidemic has spread from China to almost all countries in the world by April 1st 2020. It arrived to Colombia on March 6, 2020 from an imported case and evolved to local cases of transmission. In order to reduce the impact of the COVID-19 outbreak in Bogotá, the largest city in Colombia, a local lock-down was introduced on March 15, 2020, followed by a 
national lock-down on March 19, 2020. Colombia, like many developing nations, does not have a strong health system able to respond to a pandemic of the magnitude of the present one. Neither in terms of infrastructure, medical personnel nor in terms of logistic preparedness or technical capacities to arrange all medical needed resources. The latter is the main motivation to create a model that allows particular clinics and hospitals to estimate the number of beds and the respirators needed to attend during the peak days. Specifically, we are interested in estimating the number of patients that require Intensive Care Units-ICU care (critical), and the number of patients that require hospital care (severe), but not ICU care.

As of April 04, 2020, Colombia has only carried out 460 test per million people (https://infogram.com/, https://ourworldindata.org/covid-testing), whereas other countries such as Germany and South Korea have done over 1000 test per million people. Unfortunately, developing countries like Colombia do not have the testing facilities or production of biotechnology inputs to run the necessary number of tests required to detect all active cases of the virus. Additionally, on March 26 one of the two available machines used to run the detection tests broke, leading to a reduction on its production and causing delays in the total number of cases detected. One of the biggest concerns is that the data may not inform well how many hospital beds (and ICU beds) will be needed during the outbreak peak. In fact, one of the main caveats for this study is that the official data is very likely to be under estimated as only patients with at least one symptom or that have had contact with another detected case have been tested [1]. Moreover, we are assuming an overall probability of requiring ICU treatment, while sex, age and co-morbidity (diabetes, hypertension, acute respiratory diseases and depressed immune system) will highly determine differential probabilities, that we are not taken into account.

We implemented a SEIR model (Susceptible - Exposed - Infectious - Recovered) to forecast the number of cases in Bogotá, the largest city in Colombia and the one with the most numbers of cases to date. The model includes three different compartments of infection: Infected that require ICU care, Infected that require hospital care, but not ICU care and Infected that only require Home care. The model accounts for the effect of control strategies introduced by the government by changing the transmission rate over time. We developed a Shiny app that displays the results from the model (https://claudia-riverarodriguez.shinyapps.io/shinyappcovidclinic/). The initial parameters are the ones used in this work. However, users can change the parameters according to their needs. The Shiny app can be used as a forecast tool for specific clinics by specifying the market share (percentage) of the population corresponding to the clinic. During the outbreak some clinics should be ready to see an increase in their market share because they may have more resources, such as ICU beds and the model allows for each clinic to adjust it. The model can be used for specific cities or towns, the user only needs to change the population size, and some parameters of interest.

The results from our assumptions show that Bogotá city will need over 2000 beds, the Mayor's target number of ICUs, by the end of May 2020. By the last week of March there were 300 beds available and plans to expand it to reach 2000. This number could be well below Bogotá's needs based on our projections. 


\section{Methods}

SIR methods (Susceptible-Infected-Recovered) became widespread in the prediction of communicable diseases since its creation in the early 20th century [2]. Several authors have provided forecasting models using this method as presented in [3], but SIR models heavily rely on initial strong assumptions. SEIR models are a variation that relaxes some of those assumptions, including closed populations and account for communicable diseases that transmit in transitions, starting from the entire population (Susceptible) that incubate the disease for a period of time (Exposed) making the person infected but not infectious (I) and finally become Recovered (R) [4]. Each transition holds a rate based on what is observed from a population, that is a susceptible person gets infected at a transmission rate once in contact with an infected individual, and become exposed. Once exposed transition to infected happens at a rate that captures the inverse of the mean latent period of the disease. The final transition is recovery with permanent immunity. We chose this model to estimate bed demands per institution in Colombia separating regular and ICU (Intensive Care Units) beds, which allows a distinction for each type that reflects differential transition rates, preparedness and logistics for health providers. Similar works have been used to forecast similar needs in Europe and United States of America, but none has been done for a developing country, such as Colombia [5-7].

\subsection{Model}

We fitted a deterministic SEIR model over 12 months, for practical purposes it is important to bear in mind that policies change along this time scenario and therefore the models must be updated. The population is divided into Susceptible (S), Exposed (E), Infected (I), recovered (R), and D (death). Those Infected (I) are subdivided into three compartments: $I_{\mathrm{U}}, I_{\mathrm{NoU}}, I_{\mathrm{H}}$ which, respectively, denote infected individuals that require ICU care, infected individuals that require hospital care, but not ICU, and infected individuals that only require home care.

One implication of our model is that it does not consider events such as births, and it only considers deaths due to COVID-19. Note that we assume that patients transit from E to ICU care directly, therefore we assume that the average time from (E) to $\left(I_{\mathrm{U}}\right)$ is larger than the average time from $(\mathrm{E})$ to $\left(I_{\mathrm{NoU}}\right)$ and subsequently this is larger than the average time from $(\mathrm{E})$ to $\left(I_{\mathrm{H}}\right)$. These transitions and considerations are summarized in figure 1. We also assume that only patients in ICU die, while other infected patients recover. The total population of Bogotá is 7.4 million, but we assume an initial population size of 8 million to account for its metropolitan area because people commute to work and study daily from those surrounding towns to Bogotá Capital District.

We describe the epidemic transitions through the model 


$$
\begin{aligned}
\frac{d S}{d t} & =-\beta(t) S \frac{\left(I_{\mathrm{U}}+I_{\mathrm{NoU}}+I_{\mathrm{H}}\right)}{N} \\
\frac{d E}{d t} & =\beta(t) S \frac{\left(I_{\mathrm{U}}+I_{\mathrm{NoU}}+I_{\mathrm{H}}\right)}{N}-\left(\kappa_{\mathrm{U}}+\kappa_{\mathrm{NoU}}+\kappa_{\mathrm{H}}\right) E \\
\frac{d I_{\mathrm{U}}}{d t} & =\kappa_{\mathrm{U}} E-\gamma_{\mathrm{U}} I_{\mathrm{U}} \\
\frac{d I_{\mathrm{NoU}}}{d t} & =\kappa_{\mathrm{NoU}} E-\gamma_{\mathrm{NoU}} I_{\mathrm{NoU}} \\
\frac{d I_{\mathrm{H}}}{d t} & =\kappa_{\mathrm{H}} E-\gamma_{\mathrm{H}} I_{\mathrm{H}} \\
\frac{d R}{d t} & =(1-d) \gamma_{\mathrm{U}} I_{\mathrm{U}}+\gamma_{\mathrm{NoU}} I_{\mathrm{NoU}}+\gamma_{\mathrm{H}} I_{\mathrm{H}} \\
\frac{d D}{d t} & =d \gamma_{\mathrm{U}} I_{\mathrm{U}}+\gamma_{\mathrm{NoU}} I_{\mathrm{NoU}}+\gamma_{\mathrm{H}} I_{\mathrm{H}} \\
N & =S+I+R+D
\end{aligned}
$$

where $\kappa_{\mathrm{NoU}}=p_{\mathrm{NoU}} \kappa, \kappa_{\mathrm{U}}=p_{\mathrm{U}} \kappa$ and $\kappa_{\mathrm{H}}=p_{\mathrm{H}} \kappa$. To model the impact of interventions introduced by the government, we allow the transmission rate to be a step-wise function $\beta(t)$, with 3 steps at $t_{0}, t_{1}$ and $t_{2}$. The time $t_{0}(2020-03-06)$ corresponds to the case when no actions have been taken, $t_{1}(2020-03-29)$ is 10 days after the national lock-down was introduced and $t_{2}$ (2020-04-10) is the date when the lock-down measures are eased but other strategies such as social distance are implemented. We estimate $\beta\left(t_{0}\right)$ from the basic reproduction number such that $R_{0}=2.5$ ([8]). For $t>t_{0}$, we choose $\beta(t)$ such that $R(t) \approx 1.5$, for $t_{1} \leq t<t_{2}$ and $R(t) \approx 2$, for $t \geq t_{2}$.

The terms $p_{\mathrm{U}}, p_{\mathrm{NoU}}$ and $p_{\mathrm{H}}$ denote the probabilities that case requires ICU care, hospital non-ICU care and only home care, respectively. Note that $p_{\mathrm{U}}+p_{\mathrm{NoU}}+$ $p_{\mathrm{H}}=1$. To estimate these probabilities, we follow [9], assuming that critical cases require ICU, severe cases require hospital care, but not ICU and cases at home are mild. Their original estimates are $p_{\mathrm{U}}=0.05, p_{\mathrm{NoU}}=0.14$ and $p_{\mathrm{H}}=0.81$, but we assume that $50 \%$ of cases at home are asymptomatic and therefore not detected. We recalculated the probabilities to be $p_{\mathrm{U}}=0.026, p_{\mathrm{NoU}}=0.134$ and $p_{\mathrm{H}}=$ 0.84 The parameter $\kappa$ is the daily probability of an exposed individual becoming infected, and $\gamma_{\mathrm{U}}, \gamma_{\mathrm{NoU}}, \gamma_{\mathrm{H}}$ are the daily probabilities of the corresponding infected individuals becoming recovered or dead. The probability $d$ denotes the probability that an infected ICU individual dies. Table 1 displays the parameters of the models, their interpretation and sources The starting values for the model are based on the numbers from Colombia reported by March 28. There where 1009 active cases, 39 recovered and 17 deaths by then in the country. We assume that half of those cases correspond to Bogotá [1]. Therefore the model starts with $I_{\mathrm{U}_{0}}=p_{\mathrm{U}} * 504=13$, $I_{\mathrm{NoU}_{0}}=p_{\mathrm{NoU}} * 504=68,, I_{\mathrm{H}_{0}}=p_{\mathrm{H}} * 504=423, E_{0}=R_{0} * I=1262, D_{0}=17$, $R_{0}=39$ and $S_{0}=7999140$

\section{Results}

Figure 2 shows the results for each category the model predicts, ICU and regular hospital beds. Even all the positive measures assumed in the model, we predict that 
the peak of the epidemic can happen around 2020-07-13. During the epidemic peak, the model predicts that 22541 ICUs will be needed, and 85015 non-ICU hospital beds. We predict that the maximum number of prevalent cases will be 536673 . With the parameters in the models, the total number of deaths could reach 82536 in 6 months time.

Figure 3 displays a closer picture of those infected and the total number of deaths. We can see that the total number of infected that will need hospital care (ICU and non-ICU) are concernedly high. Additionally, figure 4 shows those infected that will need hospital care, compared to the current number of ICU beds in the city. It shows that the number of ICUs needed will be 22541 , i.e. the city has to increase its capacity by 11 times the current amount planned in order to provide care to everyone that needs it. The local authorities in Bogotá are planning to have a total of 2000 ICU beds in the city, but the current number is 300 . The number 2000 is overrun by mid May/2020, with a death toll of 351 people by then. Unfortunately, the trend keeps on increasing for the following months, which reflects the lack of preparedness for a catastrophe like the current one in Bogotá and likely in other similar developing nations.

When we double the number of days of ICU patients to recover in the model, i.e $1 / \gamma_{\mathrm{U}}=14$ days, rather than 7 , the number of ICU beds almost double in size. Moreover, if the probability of being an ICU patient $p_{U}$ is reduce, the number of beds is reduced, but even an small increase in this probability will cause a large increase in the number of ICU beds needed during the peak of the epidemic. At last, if we assume an under reporting of $20 \%$ both in the initial number of exposed, infected and deceased the 2000 beds capacity is overrun one day before.

\section{Discussion}

This document presents a prediction of the total number of ICU and regular beds the would be needed during the pandemic COVID-19 for Bogotá-Colombia. We use a SEIR model that differentiates between three types of infected patients: those who can stay at home, those in regular hospital beds and those in need of ICU treatment. With a mean incubation period of 5.2 days and mean infection period of 4 days (for patients at home), 5 days (for patients in regular hospital beds) and 7 days (for patients in ICU). The parameters assumed in the model are for a positive scenario, where the effective reproduction number during the lock-down is assumed to be 1.5, and 2 after the lock-down, when other measures are introduced. We assume that $2.6 \%$ of patients require ICU treatment, $13.4 \%$ require regular hospital beds, and the rest only require home care. The model allows for a time varying transmission rate which we use to incorporate the measures introduced by the government over the period of 1 year. The model predicts that by mid July, 2020, the city will reach the peak of the epidemic with a total of 22541 active ICU prevalent cases and 85 015 regular hospital beds needed.

By the end of May 2020, the number of patients that need hospitalization will overpass the current planned capacity set at 2000 beds for ICU beds in the city, and the death toll will reach a total of 1752 . Unpreparedness of the health system will only increase COVID-19 related and unrelated mortality, as already observed in Italy and other countries. Measures like lock-down have been used in most countries 
to diffuse the demand for health services due to COVID-19 over time, however it may be insufficient if there are not enough resources to amp the health services in developing nations, such is the case of Colombia, where the need of additional resources are a priority at this point.

Other than the intrinsic limitations of SEIR models, this prediction model does not account for age and sex distribution of the population but we plan to introduce such differences in a future version of the model with an additional mixing including the contact matrices, as the recently national population census in Colombia is available. Also, we have fitted a a model with 2 interventions: a lock-down and mitigation measures, but this can be modified later in time. Neither we accounted for regional differences that in a tropical context relate to weather and climate, because there is no evidence, to date, that the novel coronavirus could or not spread in an homogeneous pattern under certain weather conditions.

Finally, we provide a Shiny app available in https://claudia-rivera-rodriguez.shinyapps.io/shinyappcovi The original values in the app reproduce the results of this paper, but the parameters and starting values can be changed according to the users needs.

\title{
5 Conclusions
}

COVID-19 has posed too many challenges to health systems around the globe, this model is an useful tool for cities, hospitals and clinics in Colombia that need to prepare for the excess demand of services that a pandemic like this one generates. Unfortunately, the model predicts that by July capacity of the system in Bogotá will not be enough. We expect the lock-down rules strength in the future days, so the death toll is not as bad as predicted by this model.

\author{
Abbreviations \\ SEIR Susceptible-Exposed-Infected-Recovered \\ ICU Intensive Care Unit \\ COVID-19 Coronavirus disease 2019
}




\section{Declarations}

- Ethics approval and consent to participate. Not applicable

- Consent for publication. Not applicable

- Availability of data and materials. The materials(code) used and/or analysed during the current study are available from the corresponding author on reasonable request. Additionally, a shiny app is available online at https://claudia-rivera-rodriguez.shinyapps.io/shinyappcovidclinic/

- Competing interests. The authors declare that they have no competing interests

- Funding. The author(s) received no financial support for the research, authorship, and/or publication of this article.

- Authors' contributions CRR contributed to the analysis and coding of the model and the shiny app. PU contributed to model interpretation and article writing. All authors have read and approved the manuscript.

- Acknowledgements We thank the Colombia research group COVID Modelling CG for their feedback.

Competing interests

The authors declare that they have no competing interests.

\section{Author details}

${ }^{1}$ Department of Statistics, The University of Auckland, 10 Princess Street, Auckland, New Zealand. ${ }^{2}$ National University of Colombia, , , .

References

1. Instituto Nacional de Salud. https://www.ins.gov.co/. [Online; accessed April 2020]

2. Anderson, R.: The kermack-McKendrick epidemic threshold theorem. Bulletin of Mathematical Biology 53(1-2), 3-32 (1991). doi:10.1016/s0092-8240(05)80039-4

3. Murray, C.: Forecasting COVID-19 impact on hospital bed-days, ICU-days, ventilator-days and deaths by US state in the next 4 months. medRxiv (2020). doi:10.1101/2020.03.27.20043752

4. Martcheva, M.: An Introduction to Mathematical Epidemiology. Texts in applied mathematics, vol. Volume 61. Springer, ??? (2015). OCLC: 934647894

5. Li, R., Rivers, C., Tan, Q., Murray, M., Toner, E., Lipsitch, M.: The demand for inpatient and ICU beds for COVID-19 in the US: lessons from chinese cities. medRxiv (2020). doi:10.1101/2020.03.09.20033241

6. Massonnaud, C., Roux, J., Crépey, P.: COVID-19: Forecasting short term hospital needs in france. medRxiv (2020). doi:10.1101/2020.03.16.20036939

7. Zhang, T., McFarlane, K., Vallon, J., Yang, L., Xie, J., Blanchet, J., Glynn, P., Staudenmayer, K., Schulman, K., Scheinker, D.: A model to estimate bed demand for COVID-19 related hospitalization. medRxiv (2020). doi:10.1101/2020.03.24.20042762

8. Kucharski, A.J., Russell, T.W., Diamond, C., Liu, Y., Edmunds, J., Funk, S., Eggo, R.M., Sun, F., Jit, M., Munday, J.D., Davies, N., Gimma, A., [van Zandvoort], K., Gibbs, H., Hellewell, J., Jarvis, C.I., Clifford, S., Quilty, B.J., Bosse, N.I., Abbott, S., Klepac, P., Flasche, S.: Early dynamics of transmission and control of covid-19: a mathematical modelling study. The Lancet Infectious Diseases (2020). doi:10.1016/S1473-3099(20)30144-4

9. Wu, Z., McGoogan, J.M.: Characteristics of and Important Lessons From the Coronavirus Disease 2019 (COVID-19) Outbreak in China: Summary of a Report of 72314 Cases From the Chinese Center for Disease Control and Prevention. JAMA (2020). doi:10.1001/jama.2020.2648

10. Liu, Y., Gayle, A.A., Wilder-Smith, A., Rocklöv, J.: The reproductive number of COVID-19 is higher compared to SARS coronavirus. Journal of Travel Medicine 27(2) (2020). doi:10.1093/jtm/taaa021

11. Zhang, J., Litvinova, M., Wang, W., Wang, Y., Deng, X., Chen, X., Li, M., Zheng, W., Yi, L., Chen, X., Wu, Q., Liang, Y., Wang, X., Yang, J., Sun, K., Longini, I.M., Halloran, M.E., Wu, P., Cowling, B.J., Merler, S., Viboud, C., Vespignani, A., Ajelli, M., Yu, H.: Evolving epidemiology and transmission dynamics of coronavirus disease 2019 outside hubei province, china: a descriptive and modelling study. The Lancet Infectious Diseases (2020). doi:10.1016/s1473-3099(20)30230-9

12. Liu, T., Hu, J., Xiao, J., He, G., Kang, M., Rong, Z., Lin, L., Zhong, H., Huang, Q., Deng, A., Zeng, W., Tan, X., Zeng, S., Zhu, Z., Li, J., Gong, D., Wan, D., Chen, S., Guo, L., Li, Y., Sun, L., Liang, W., Song, T., He, J., Ma, W.: Time-varying transmission dynamics of novel coronavirus pneumonia in china. bioRxiv (2020) doi:10.1101/2020.01.25.919787

13. Prem, K., Liu, Y., Russell, T.W., Kucharski, A.J., Eggo, R.M., Davies, N., Jit, M., Klepac, P., Flasche, S., Clifford, S., Pearson, C.A.B., Munday, J.D., Abbott, S., Gibbs, H., Rosello, A., Quilty, B.J., Jombart, T., Sun, F., Diamond, C., Gimma, A., van Zandvoort, K., Funk, S., Jarvis, C.I., Edmunds, W.J., Bosse, N.I., Hellewell, J.: The effect of control strategies to reduce social mixing on outcomes of the COVID-19 epidemic in wuhan, china: a modelling study. The Lancet Public Health (2020). doi:10.1016/s2468-2667(20)30073-6 
14. Lin, Q., Zhao, S., Gao, D., Lou, Y., Yang, S., Musa, S.S., Wang, M.H., Cai, Y., Wang, W., Yang, L., He, D.: A conceptual model for the coronavirus disease 2019 (COVID-19) outbreak in wuhan, china with individual reaction and governmental action. International Journal of Infectious Diseases 93, 211-216 (2020). doi:10.1016/j.ijid.2020.02.058

15. van den Driessche, P.: Reproduction numbers of infectious disease models. Infectious Disease Modelling 2(3), 288-303 (2017). doi:10.1016/j.idm.2017.06.002

16. Diekmann, O., Heesterbeek, J.A.P., Roberts, M.G.: The construction of next-generation matrices for compartmental epidemic models. Journal of The Royal Society Interface 7(47), 873-885 (2009). doi:10.1098/rsif.2009.0386 
Table 1 Parameters and definition for model (1)

\begin{tabular}{|c|c|c|c|}
\hline Symbol & Definition & Value & Source \\
\hline$\beta(t)$ & Transmission rate & $\begin{array}{l}\text { Stepwise func- } \\
\text { tion }\end{array}$ & {$[8,10]$} \\
\hline$\kappa$ & $\begin{array}{l}\text { Daily probability of an exposed individ- } \\
\text { ual becoming infected: } \kappa=1 / \alpha \text {, with } \alpha \\
\text { being the mean incubation period }\end{array}$ & $1 / 5.2$ & [11] \\
\hline$p_{\mathrm{U}}$ & Probability of patient being ICU & 0.026 & [9] \\
\hline$p_{\text {NoU }}$ & $\begin{array}{l}\text { Probability of patient being in Hospital, } \\
\text { but not ICU }\end{array}$ & 0.134 & [9] \\
\hline$p_{\mathrm{H}}$ & $\begin{array}{l}\text { Probability of patient being mild/at } \\
\text { home }\end{array}$ & 0.84 & [9] \\
\hline$\gamma_{U}$ & $\begin{array}{l}\text { Daily probability that an infected indi- } \\
\text { vidual in ICU recovers, when the mean } \\
\text { infection period is } b_{U}, \gamma_{U}=1 / b_{U}\end{array}$ & $1 / 7$ & [11-13] \\
\hline$\gamma_{\text {NoU }}$ & $\begin{array}{l}\text { Daily probability that an infected indi- } \\
\text { vidual in Hospital, but no ICU recovers, } \\
\text { when the mean infection period is } b_{\text {NoU, }}\end{array}$ & $1 / 5$ & [11-14] \\
\hline$\gamma_{\mathrm{H}}$ & $\begin{array}{l}\gamma_{\mathrm{NoU}}=1 / b_{\mathrm{NoU}} \\
\text { Daily probability that an infected indi- } \\
\text { vidual in Hospital, but no ICU recovers, } \\
\text { when the mean infection period is } b_{\mathrm{H}} \text {, } \\
\gamma_{\mathrm{H}}=1 / b_{\mathrm{H}}\end{array}$ & $1 / 4$ & [11-14] \\
\hline$d$ & $\begin{array}{l}\text { Probability of dying given that patient is } \\
\text { in ICU }\end{array}$ & 0.50 & [9] \\
\hline
\end{tabular}




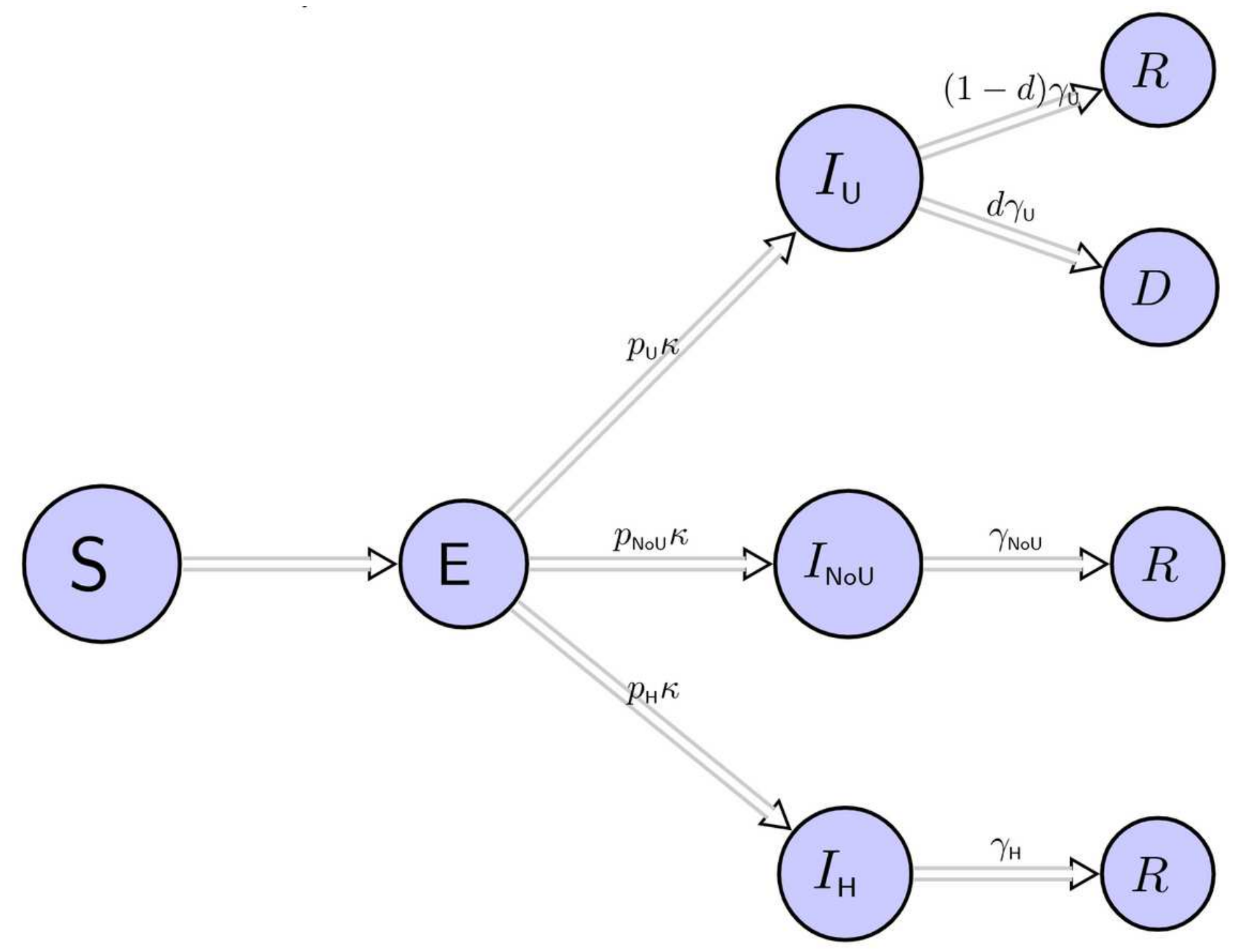

\section{Figure 1}

We divided the population into susceptible (S), exposed (E), infected ICU (IU), infected in hospital but not ICU (INoU ), infected that require only home care $(\mathrm{IH})$, recovered $(R)$ and dead subjects (D) individuals. Infected subjects are IU; INoU or IH with probabilities $\mathrm{pU}$; pNoU and $\mathrm{pH}$ respectively. The term 1/KV is the mean incubation period and $1 / \mathrm{YU} ; 1 / \mathrm{YNoU}, 1 / \mathrm{YH}$ are the daily probabilities that the respective patients recover. $d$ is the probability of death for ICU patients. 


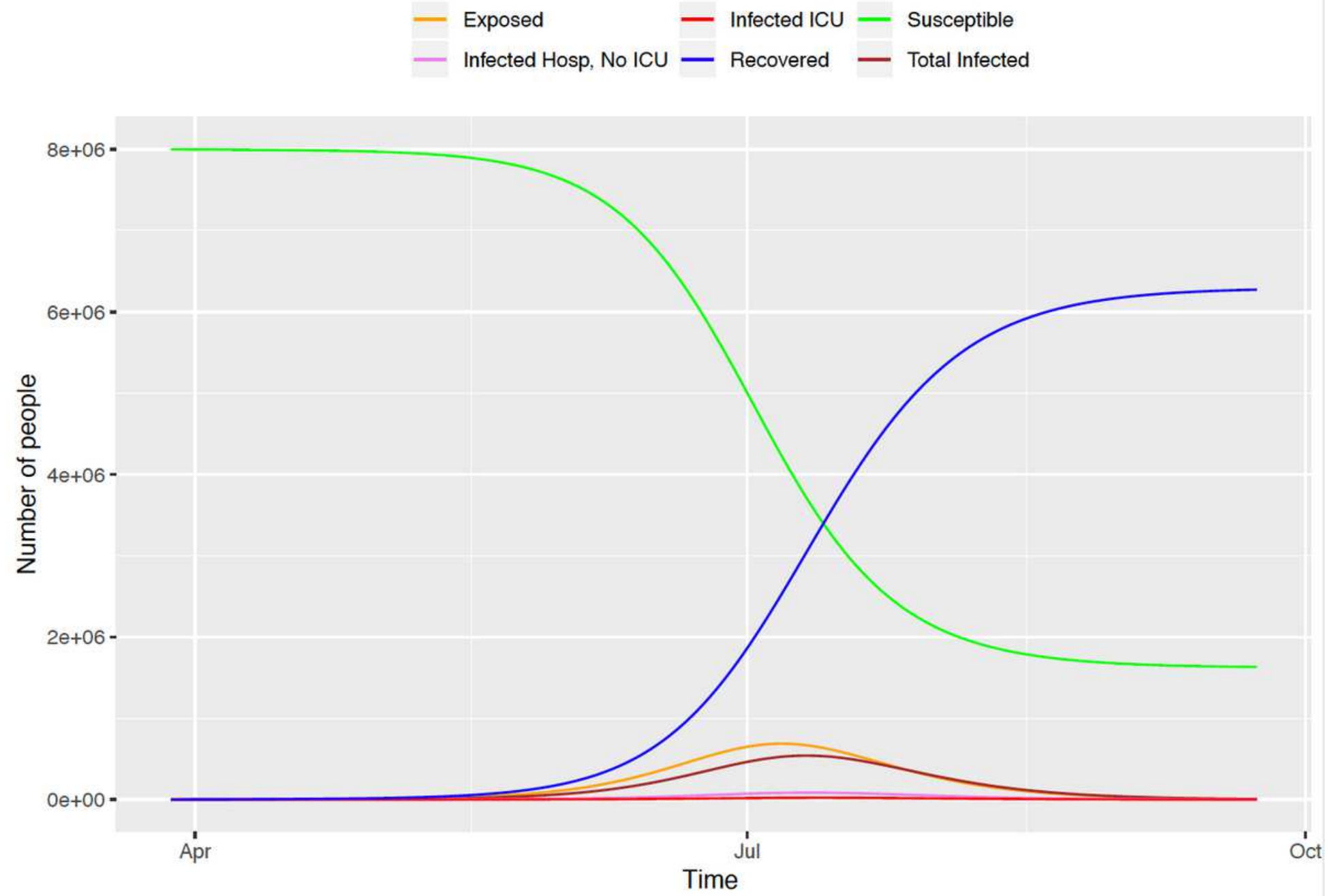

Figure 2

Progress of the epidemic using model (1) 


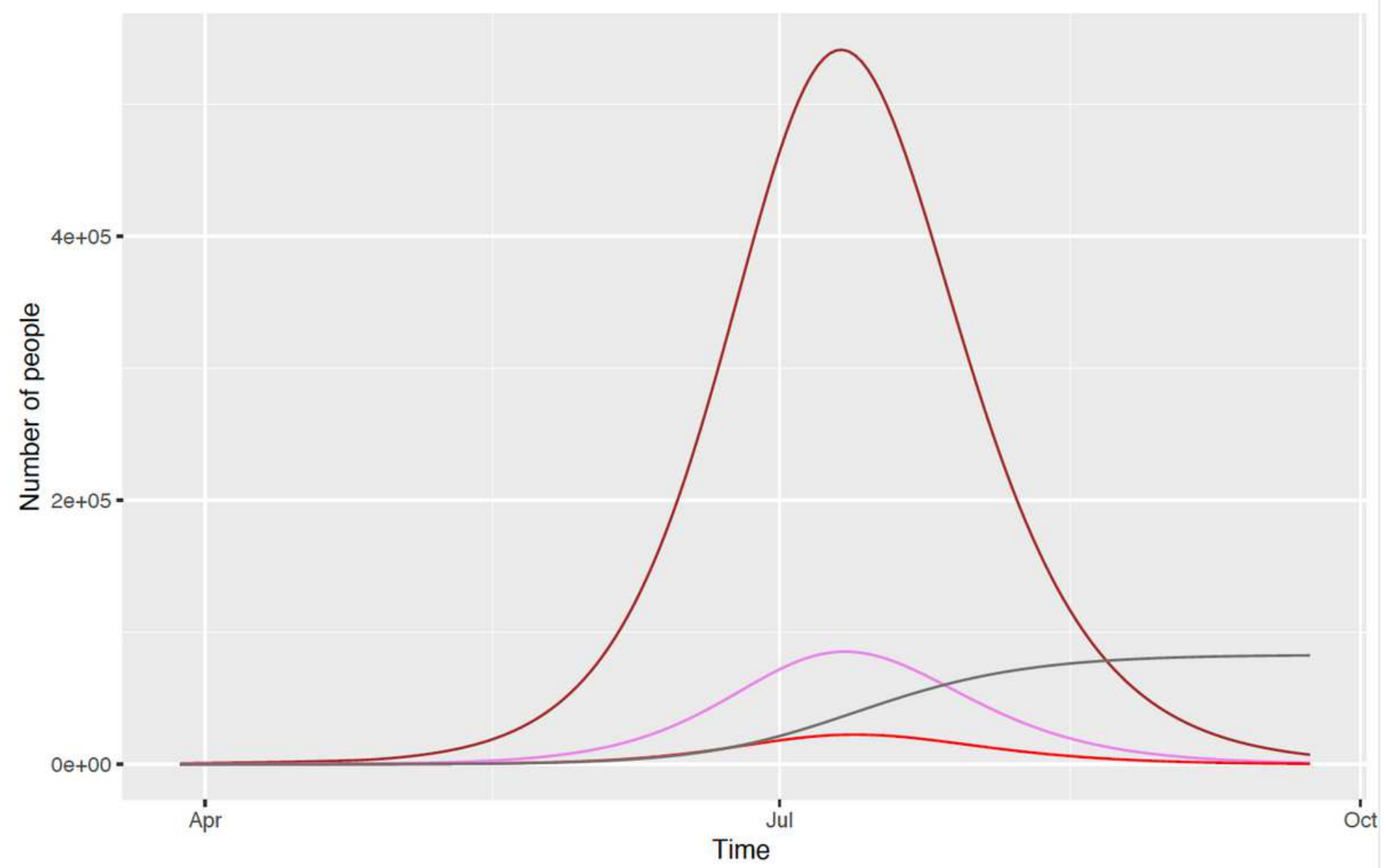

\section{Figure 3}

Progress of the epidemic - infected and deaths, results from model (1) 
— Current ICU Beds — Infected Hosp, No ICU — Infected ICU

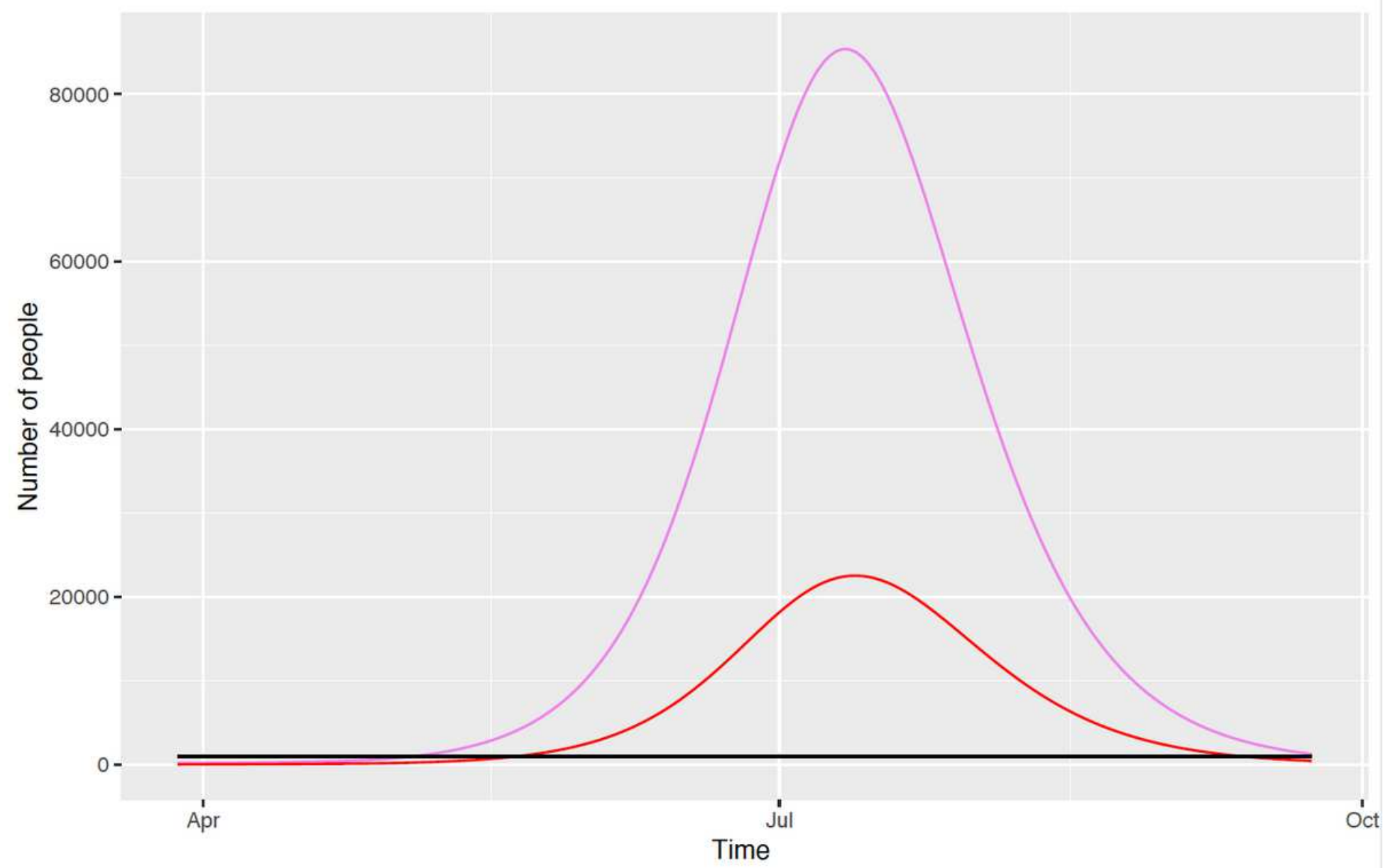

\section{Figure 4}

Progress of the epidemic - Infected that require ICU and infected that require regular hospital beds, results from model (1)

\section{Supplementary Files}

This is a list of supplementary files associated with this preprint. Click to download.

- PUBHD2002036R1appendix.pdf 